\title{
An Evidence-Based Framework to Optimise Social Impact in Astronomy for Development
}

\author{
Eli Grant \\ IAU Office of Astronomy for Development, Cape Town, South Africa \\ email: eg@astro4dev.org
}

\begin{abstract}
Astronomy for development projects conceive of development in very broad terms and seek to affect a wide range of social outcomes. The histories of education, development economics and science communication research indicate that positive social impacts are often difficult to achieve. Without a scientific approach, astronomy's potential as a tool for development may never be realised nor recognised. Evidence-informed project design increases the chances of a project's success and likely impact while reducing the risk of unintended negative outcomes. The IAU Office of Astronomy for Development (OAD) Impact Cycle is presented here as a possible framework for integrating evaluation and evidence-based practice in global Astronomy outreach and education delivery. The suggested framework offers a way to gradually accumulate knowledge about which approaches are effective and which are not, enabling the astronomy community to gradually increase its social impact by building on its successes.
\end{abstract}

Keywords. outreach, science communication, education, development, evaluation, evidencebased practice

\section{Background}

The International Astronomical Union's (IAU) Strategic Plan 2010-2020 Astronomy for Development (2012) set out the IAU's vision of how astronomy could be used as a tool to achieve a range of development objectives and resulted in the establishment of the IAU Office of Astronomy for Development (OAD) in 2011. In these proceedings, Govender describes the work done by the OAD to expand the reach and capacity of astronomy for development projects. Since the OAD's establishment, thousands of students and members of the public have been exposed to Astronomy through a diverse range of projects seeking to realise the plan's aims.

The ability to reach people is a prerequisite for social impact. Exposure and impact are not, however, synonymous. Some interventions are more effective than others. Not all individuals who have been exposed to astronomy have been effectively engaged or learned what the project intended them to learn. From a more sceptical, scientific point of view each project (and the IAU Strategic Plan itself) is an idea, essentially a hypothesis, about a specific way in which astronomy might be used for positive social impact. This is how the IAU's Strategic Plan was deliberately conceived, allowing for grassroots innovation and experimentation. Unless we can test these hypotheses, however, we do not know which projects have been most and least effective. Measuring project impacts would help to ensure that limited resources are directed to the most cost-effective projects: that is, activities that produce the largest impacts for the largest number of people for the least amount of money. 


\section{Good Intentions and Unintended Consequences}

Human beings are complex and embedded in complex systems. For some natural scientists, social complexity suggests that scientific measurement and testing is not possible. However, methodological and computing advances across multiple disciplines over the past century have provided valuable tools for detecting signals amidst the randomness and noise. There are also regularities in how human beings tend to respond. Indeed, human and social complexity is the very reason that it is imperative to test project impact $†$. A few examples of unintended consequences from astronomy for development projects include loss of interest in astronomy from those previously interested; loss of self-confidence and interest in science when educational activities are mismatched in pace or content to some students' baseline skills and knowledge; and the perpetuation of negative social norms in projects that bring together disadvantaged youth. The probability of negative unintended consequences can be reduced by learning from other fields working to effect positive social impact and adopting a more scientific, evidence-informed approach.

\section{Methods for Measuring Project Impact}

There are three key challenges for determining which projects 'work', which do not and which are most likely to work in a new context. These are: measuring target outcomes, measuring a project's causal effects and then predicting likely effects in a new context. Not all social impacts are measurable. Many astronomy for development projects, for example, focus on inspiring their audiences. Inspiration is not readily measurable. However, observable changes must occur at some point for a project to be considered useful for development. Current global development priorities can be found in the Sustainable Development Goals (SDGs), agreed by the United Nations this year after extensive surveys and consultations with millions of citizens, experts, governments and development organisations across the world. These goals are the outcome of a long consensus-building process focused on identifying which aspects of development matter most. Each goal is associated with a list of targets or indicators: these are observable and measurable $\ddagger$. Examined in relation to the SDGs, inspiration might be seen as a key stepping stone towards achieving a multitude of development goals (such as improvements in education quality) rather than a final goal. Many astronomy for development projects target multiple, observable, development-related outcomes alongside invisible or internal psychological processes. Examples of observable and measurable outcomes typically targeted by astronomy for development projects include improved or increased learning outcomes; participation in science classes; scientific literacy; selection of science majors by undergraduates; enrolment in science, physics or astronomy courses or post-graduate studies; and research output.

Measuring the causal effects of project activities on observable outcomes is the second challenge. Drawing from medical science and economics, a project's causal impacts and/or key components can be tested using experimental designs. In these applications, experimental designs are known as randomised controlled trials. Participants are randomly assigned to the experimental condition (e.g. an innovative astronomy outreach activity)

$\dagger$ See: Macintyre, S. \& Petticrew, M. (2000) Good intentions and received wisdom are not enough. Journal of Epidemiology and Community Health, 54, 802-803 and Dishion, T. J., McCord, J. \& Poulin, F. (1999) When interventions harm: Peer groups and problem behavior. American Psychologist, 54, 755-764.

$\ddagger$ See the International Council of Science Union's (ICSU) report Review of Targets for the Sustainable Development Goals: A Science Perspective, available from http://www.icsu.org/publications/ 
or to an alternative (e.g. no activity or a standard astronomy outreach activity). Because assignment to one condition or the other is random, it is statistically independent of outcome: the two samples are drawn from the same population, with intervention exposure known to be the only thing distinguishing them. The average outcome in the comparison condition is thus known to offer a measure of what the average outcome for the experimental sample would have been. In the absence of random assignment, it is often impossible to know what caused what. For example, if a high proportion of students who attend a summer school enrol in astronomy postgraduate courses, is that because the school motivated and engaged them? Or is that because only those students who were most interested (and already intending to continue with astronomy) were the ones who were most likely to apply and be best-prepared for the school? Would they have otherwise enrolled in a different areas of science? Or did the summer school actually increase the total number of science post-graduates in the target population? Because they are often over-subscribed, summer schools and workshops are particularly well-suited to impact evaluation using random assignment.

A particularly promising and often low-cost approach for helping projects to learn from one another is to randomly assign participants to experience different versions of a project. An excellent example of this approach is offered by Buck (2013), who randomly assigned post-secondary students to visualisations of dark matter in a planetarium shown in three different colours. She found that those exposed to the 'standard' visualisation (dark matter depicted in white so that it stands out from a dark blue background), were four times less likely to correctly identify dark matter in a post-exposure test than participants who were exposed to visualisations where dark matter was darker than the background $\dagger$. Findings from this simple experiment can now be used to improve learning outcomes amongst planetarium attendees.

Readers interested in learning more about research and project evaluation methodologies are referred to (1) the MIT Poverty Action Lab (JPAL), who conduct randomised controlled trials of international development projects including those in primary and secondary eduction and provide both data from these experiments and extensive resources on evaluation methods themselves: www. povertyactionlab.org/education (2) Nobel prize-winning economist Angus Deaton's more in-depth explanation of the statistical theory underpinning randomised controlled trials and ongoing debates as to whether project mechanisms rather than whole projects should be tested: Deaton, A. (2009) Instruments of Development: Randomization in the Tropics, and the Search for the Elusive Keys to Economic Development. NBER Working Paper,14690. For examples of how evidence and evaluation research have been used to inform the design and implementation of projects in related fields see Yeager and Walton (2011) Social-Psychological Interventions in Education: Theyre Not Magic. Review of Educational Research, 267-301.

\section{The OAD Impact Cycle to Support Astronomy-for-Development}

Simple experiments like these can advance our understanding of how to achieve social impact more effectively. Through co-ordination and collaboration across the education, outreach and development communities, the OAD hopes that more experiments will be conducted and fed into a shared database that can be used to make evidence-based decisions about 'what works'. The aim is to create a positive feedback cycle through which projects can draw on research that has already been conducted to inform their project

$\dagger$ Buck, Z. (2013) The Effect of Color Choice on Learner Interpretation of a Cosmology Visualization. Astronomy Education Review, 12, 1, 010104. 


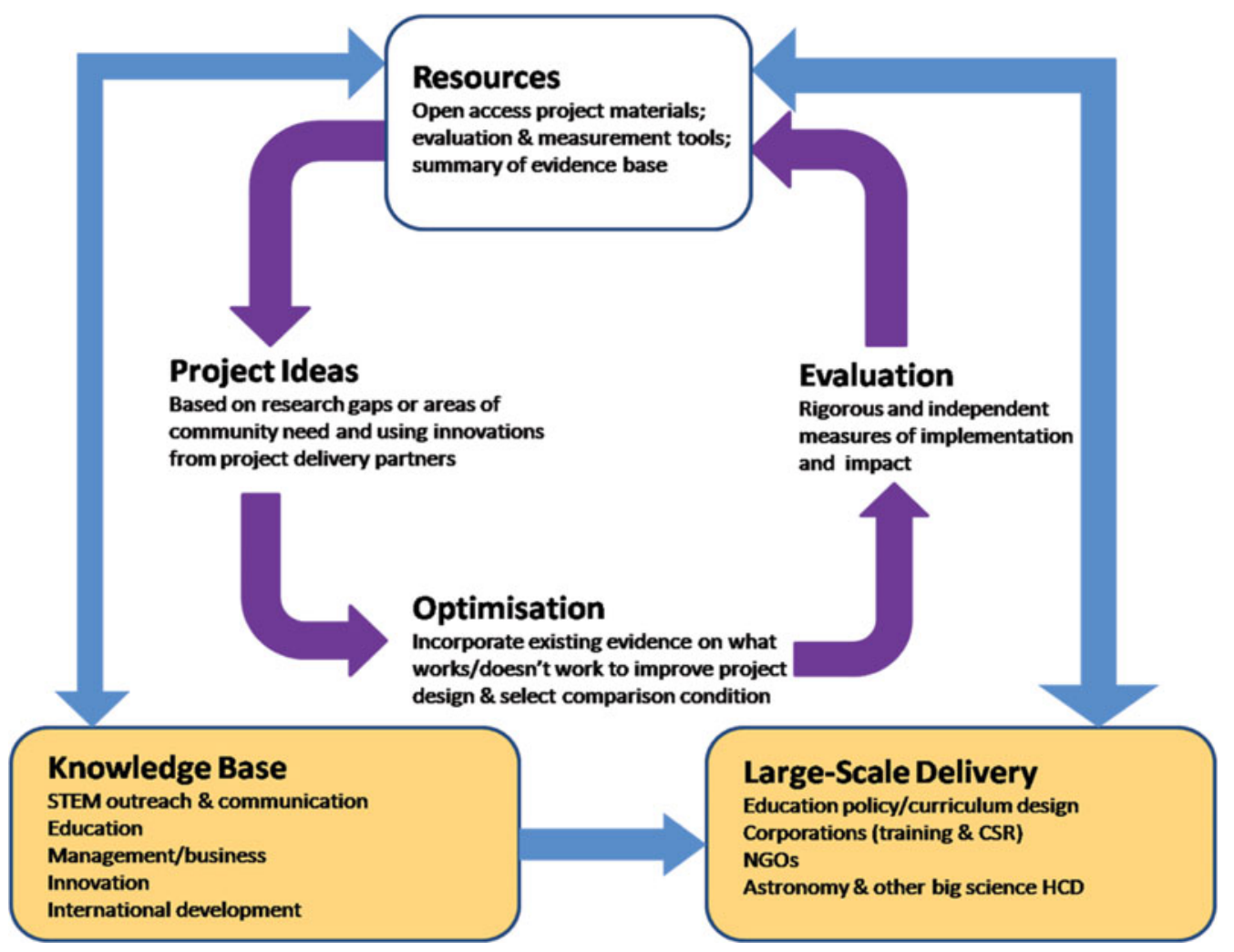

Figure 1. OAD Impact Cycle for Project Evaluation and Optimisation

designs; test creative new ideas, innovations and adaptations, including implementation in a new context or country; and then contribute their own results to a gradually accumulating knowledge base. This framework is referred to as the OAD 'Impact Cycle' (see Figure 1).

Resources will include systematic reviews $\dagger$ of relevant evidence (e.g. educational techniques and international development programmes); best-practice guidelines; standardised and validated outcome measures (e.g. of scientific and statistical literacy) and evaluation tools to assist in the design and implementation of project evaluations and outcome measurement. The Ideas stage refers to support for creative innovations or projects that members of the astronomy community come up with for how astronomy might be used for development. Project ideas will ideally be informed by Resources or developed in response to specific development-related problems or needs. Optimisation refers to a stage of refinement and improvement of project ideas, informed by evidence provided in the Resources of what has already been tried, what works and what does not. The Ideas and Optimisation phases provide mechanisms through which innovations and ideas can more easily build upon, rather than reinvent, previous work. Drawing from evidence will allow new ideas to maximise their likelihood of success and reduce their risk of unintended negative consequences. Finally the Evaluation phase involves embedding impact evaluation designs of entire projects or of specific innovative components within projects,

$\dagger$ See for example Greenhalgh, T. (1997) How to read a paper. Papers that summarise other papers (systematic reviews and meta-analyses). BMJ, 13, 315(7109):672-5. 
to complete the cycle and contribute new evidence of 'what works' to the communities' resources.

\section{Conclusion}

The OAD Impact Cycle offers a framework for accumulating scientific evidence from across contexts and countries to support efforts to understand the variability and localisation of specific types of projects' impacts. The Impact Cycle seeks to support the astronomy community in drawing from resources from other disciplines, including science communication, education and development efforts across fields of science, technology, engineering and mathematics. The Impact Cycle also offers a way through which the astronomy community can contribute to global development efforts by participating in cross-disciplinary research on what works. 